\title{
Zur Kenntniss der Überwallungsharze
}

von

Max Bamberger.

Aus dem Laboratorium fuir allgemeine und analytische Chemie an der k. k. technisehen Hochschule in Wien.

Mit 1 Textfigur:

(Vorgelegt in der Sitzung am 16. Juli 1891,)

Nachdem auf Anregung des Herrn Prof. Benedikt im chemischen Laboratorium der technischen Hochschule das Verhalten der Harze bei den quantitativen Reactionen zuerst von v. Schmidt und Erban ${ }^{1}$ studirt, und deren Angaben sodann von mir durch die Bestimmung des Methoxylgehaltes ${ }^{2}$ ergänzt worden waren, konnte auf Grund der erhaltenen Daten an eine eingrehendere wissenschaftliche Untersuchung jener Harze gegangen werden, welche bei diesen Reactionen besonders auffallende Zahlen ergaben.

Ich habe auf Wunsch des Hern Prof. Benedikt zunächst das Studium der Überwallungsharze der einheimischen Coniferen tibernommen, welches desshalb interessante Resultate zu liefern versprach, weil ich in einem derselben, dem Überwallungsharze der Schwarzföhre (Pinus Laricio Poir.) einen auffallend hohen Methoxylgehalt entdeckt hatte, während andere Coniferenharze, z. B. Colophonium, das Scherrharz, die Harze von Pinus Taeda und Pinus halepensis kein Methoxyl enthalten.

Über Veranlassung des Herm Prof. J. Wiesner wurden zwei Überwallungsharze von Herrn Dr. F. Krasser, Assistent am pflanzenphysiologisehen Institute der hiesigen Universität,

1 Monatshefte für Chemie, VII, 655.

2 Ebenda, XI, 84. 
einer näheren Untersuchung unterzogen, wofiu ihm hier der wärmste Dank ansgedrickt werde. Dr. F. Krasser schreibt diesbeziiglich:

„Bei der naturhistorischen Charakterisirung der beiden Harzproben ergab sich, dass das eine sich als typisches Überwallungsharz der Schwarzföhre (Pinus Laricio Po ir.) erwies, das andere als Überwallungsharz der Fichte (Picea vulgaris Link) anzusehen sei. Das Überwallungsharz der Schwarzföhre wurde schon 1869 von Wiesner entdeckt und in einem seiner für die technische Waarenkunde grundlegenden Werke ${ }^{1}$ beschrieben. Behufs Vergleichung mit dem erst zu charakterisirenden Überwallungsharz der Fichte erlaube ich mir diese Beschreibung, in einigen Punkten ergänıt, wiederzugeben, daran soll sich die naturhistorische Charakteristik des Überwallungsharzes der Fichte anschliessen.

\section{a) Das Überwallungsharz der Schwarzföbre. ${ }^{2}$}

Die vorliegenden Stückchen baben die Gestalt kleiner Rollkieselsteine, im Durchmesser meist $1-2 \mathrm{~cm}$. Die Oberfläche ist durch Verwitterung matt geworden. Die Bruchflächen frisch aufgebrochener Stücke sind von gelblicher Farbe und werden an der Atmosphäre roth mit einem Stich ins Violette. Das gepulverte Überwallangsharz wird schon nach einigen Stunden pirsichblühroth. Das Überwallungsharz repräsentirt sich als dichte homogene Masse, welche von kleinen bis stecknadelkopfgrossen, zusammenhanglosen Hohlräumen durchsetzt ist. In die amorphe Grundsubstanz sind zahlreiche doppeltbrechende Krystalle eingelagert. Reichlich sind schief rhombische Tafeln vorhanden, welche namentlich nach kurzer Einwirkung von Terpentinöl oder absolutem Alkohol deutlich hervortreten. Die ausserdem in der Grundmasse vorhandenen ausserordentlich zahlreichen kleinen, wetzsteinförmigen Krystalle treten vornehmlich zwischen gekreuzten Nicols dentlich in Erscheinung, wobei sie in verschiedenen Farben

1 Wiesner, "Die technisch verwendeten Gummiarten, Harze und Balsame". Erlangen 1869, 114 und "Die Rohstoffe des Pflanzenreiches", Leipzig 1873, 100.

2 Die mitgetheilte Beschreibung fusst anf der von Wiesner 1. c. gegebenen. 
aufleuchten. An den Kanten sind die Harzsplitter durchscheinend, der Bruch ist flach muschelig, der Glanz als Fettglanz, bernsteinartig zu bezeichnen. Die Dichte ist grösser als die des Wassers und geringer als die der concentrirten Schwefelsäure. Tenacität: spröde bis milde. Strich: weiss. Der Geruch ist angenehm and erinnert an die Zimmtsäure führenden Harze. Sehr deutlich tritt der aromatische Geruch beim gepulverten Harz auf, welches iibrigens auch einen an Buttersäure erinnernden Geruch besitzt. Geschmack milde, aromatisch, nicht bitter. Organische Einschlüsse fanden sich nicht vor.

\section{b) Das Überwallungsharz der Fichte.}

Knollenförmige, nnregelmässig gestaltete Stiickchen rerschiedener Grösse, 1--3 cm im Durchmesser, mit höckeriger, durch Verwitterung matter Oberfläche.

Die frischen Bruchflächen sind bei den festesten Stückchen gelblich und weiss gesprenkelt und werden an der Atmosphäre - wie beim Überwallungsharze der Schwarzkiefer - roth mit einem Stich ins Violette. Bei den meisten Stückchen erscheint jedoch die Bruchfläche marmorirt, der conglomeratartigen Structur des Harzes entsprechend, welches sichtlich aus weissen, gelben bis braunen Massen zusammengesetzt ist. Das gepulverte Harz wird auch nach Tagen nicht pfirsichblühroth, sondern licht zimmtbraun. Die dichtesten Stiicke sind der Structur nach homogen, hie und da von sehr kleinen zusammenhanglosen Hohlräumen durchsetzt, während andere Stücke fast körnige Struetur aufweisen. Homogene und körnige Structur sind oft an einem Stücke in seinen verschiedenen Partien zu beobachten. In die amorphe Grundmasse sind theils tafelförmige, theils kleine wetzsteinförmige Krystalle einge]agert. Sie sind zahlreich in den dichten homogenen Partien, treten fast gänzlich zurtick in denjenigen, wo das Harz in Folge reichlichen Gehaltes an ätherischem Öle geschmeidig ist. Harzbruchstiicke sind an den Kanten durchscheinend. Frische Bruchflächen besitzen Fettglanz. Der Bruch ist als flach muschelig bis erdig zu bezeichnen. Dichte grösser als die des Wassers, geringer als die der concentrirten Sehwefelsäure. Tenacität: milde bis geschmeidig. Strich: weiss. Geruch lohartig, erinnert weder an Zimmtsäure, noch an Buttersäure; 
namentlich am Harzpulver gut wahrzunehmen. Geschmack milde, etwas aromatisch, bäufig ingwerartig, nicht bitter.

Organische Einschliisse. Einzelne von den Harzstucken waren mit Rindenfragmenten im Zasammenhange. Die Art und Weise des Zusammenhanges liess das fragliche Harz als Überwallungsharz erkennen. Die anatomische Untersuchung der erwähnten Rindenfragmente lehrte, dass dieselben von Picea vulgaris Link herstammen. Diese Entscheidung wurde dadurch erleichtert, dass als Stammptlanze des Harzes nur Tanne, Lärche, Föhre und Fichte in Betracht kamen. Die anatomische Untersuchung der Rinden von Tanne, Lärche und Fichte wurde bekanntlich schon v. Höhnel (1880) ${ }^{1}$ dargelegt, während Möller ${ }^{2}$ auch die Föhre aufgenommen hat. Da das zu bestimmende Rindenfragment in der secundären Rinde Steinzellen besass, bei Pinus aber die secundäre Rinde gar keine sklerotischen Elemente enthält, ${ }^{3}$ so konute die Identificirung der fraglichen Rinde unter Berücksichtigung der Angaben v. Höhnel's nicht schwer fallen; sie war dann schon an dem gänzlichen Feblen von Spicularzellen als Fichtenrinde zu erkennen."

Das Überwallungsharz der Schwarzföhre.

Eine grössere Menge des Überwallungsharzes der Schwarzföhre verdanke ich der Güte des Herrn C. Mitterer, Besitzer einer Mühle und Harzsiederei in Weissenbach an der Triesting. Ich erlaube mir hier an dieser Stelle, Hern Mitterer für die Besorgung dieses nicht leicht zu beschaffenden Harzes meinen wärmsten Dank auszusprechen.

Der Schmelzpunkt des Überwallungsharzes liegt bei circa $100^{\circ}$. Es löst sich leicht in Alkohol, Äther, Methylalkohol, Eisessig, Amylalkohol, Aceton, theilweise in Benzol, Cbloroform, Schwefelkohlenstoff, Terpentinöl, fast nicht in Petroleumäther.

Um den Eiufluss des Lichtes und der Luft auf das Harz zu ermitteln, wurde es im Dunkeln gepulvert, worauf nach kurzer Zeit intensive Rothfärbung eintrat. Lässt man das gelbe Harz in die Barometerleere aufsteigen, so ist nicht die geringste Roth-

1 Die Gerberrinden. Berlin 1880, S. 41 nnd 42.

2 Anatomie der Baumrinden. Berlin 1882.

3 Möller, 1. c. S. 426. 
färbung zu bemerken, selbst wenn das Harz lange dort bleibt. Setzt man das rothe Harz längere Zeit dem Einflusse der Luft aus, so geht die rothe Farbe in eine lichtbräunliche tiber.

Bei der Destillation des Harzes im Vacuum geht eine bräunlich gefärbte, dickflüssige Masse uiber, welche noch nicht näher untersucht worden ist. Versetzt man eine alkoholisehe Lösung des Harzes mit einer Bleizuckerlösung, so erhält man einen sebr reichlichen gelben Niedersclilag. Das Harz gibt eine sehr intensive Phloroglucinreaction.

Die Säure- und Jodzahl des Harzes wurde nach der Methode von Schmidt und Erban' bestimmt. Die Köttstorfer'sche Zahl kounte nicht bestimmt werden, da die alkalische Lösung des Harzes so dunkel gefärbt war, dass an ein Titriren nicht gedacht werden konnte.

$$
\underbrace{\text { Säurezahl }}_{116 \cdot 6} \quad \frac{\text { Jodzahl }}{51 \cdot 9 .}
$$

Die Methylzahlen wurden mit dem Methoxylbestimmungsapparat von Benedikt und Grïssner vorgenommen.

Methylzahlen.

$\begin{gathered}\text { Harz von } \\ \text { Prof. Höhnel }\end{gathered}$
$\begin{gathered}49 \cdot 6 \\ 50 \cdot 9\end{gathered}$

Harz von

Weissenbach

$54 \cdot 8$

$54 \cdot 1$.

Ein Vorversuch ergab die Beobachtung, dass, wenn man das Har' längere Zeit mit Wasser kocht, die Lösung vom geschmolzenen Harze abfiltrirt und mit $\ddot{A}$ ther ausschüttelt, bei dessen Verdunsten eine ziemlich reichliche Menge eines krystallinischen Rückstandes bleibt.

Zur Darstellnng einer grösseren Menge dieses Rückstandes wurden circa $3 \mathrm{~kg}$ des Harzes aufgearbeitet. Es erwies sich als vortheilhafter, das Harz zuerst in Alkohol aufzulösen und diesen dann durch Einleiten von Wasserdampf zu entfernen, als das Auskochen mit Wasser direct vorzunehmen. Die obige Quantität

1 Monatshefte fiur Chemie, VII, 655. 
Harz wurde mit etwa $9 l 70 \%$ igen Alkohol iibergossen und einige Zeit stehen gelassen, wobei der grösste Theil des Harzes in Lösung ging. In der abfiltrirten, alkoholisehen Flïssigkeit wurde nun in mehreren Partien der Alkohol durch Einleiten von Wasserdampf entfernt. Das Harz schied sich bei dieser Operation am Boden des Gefässes als geschmolzene Masse ab. Die über dem Harze befindliche wässerige Lösung $A$ wurde abfiltrirt nnd einige Zeit stehen gelassen, wobei sich aus ihr eine grössere Menge eines harzartigen Körpers ausschied, welcher anfangs weich, später zu einer von zahlreichen Krystallen durchsetzten Masse erstarte. Auf die Untersuchung dieser Masse wurde vorderhand nur insoweit eingegangen, als die Methylzahl bestimmt wurde, und zwar betrug dieselbe $60 \cdot 4$, war also grösser als die des urspriinglichen Har'zes.

\section{Aufarbeitung von $\boldsymbol{A}$.}

Aus der wässrigen Lösung sehieden sich nach einigen Tagen reichliche Mengen von krystallinischen, weissen Krusten ab. Dieselben wurden anfangs abfiltrirt, im Verlaufe der Untersuchung zeigte es sich jedoch, dass dies nicht notbwendig ist, dass es vielmehr geniigt, die Fliissigkeit sammt den Krusten dreimal mit Äther auszuschtutteln. Beim Abdestilliren desselben hinterblieb im Destillationskolben eine reichliche Menge eines schön krystallisirten, gelblich gefärbten Rückstandes. Diesel Rückstand wurde in $95 \%$ igem Alkohol gelöst; die concentrirte Lösung erstarte sehr bald zu einem krystallinischen Brei, welcher durch Absaugen von der Mutterlauge getrennt wurde. Diese Operation mehreremale vorgenommen, lieferte eine gelblichweisse Masse, welche nun mit heissem Wasser, in dem sie ziemlich leicht löslich ist, umkystallisirt wurde. Es schieden sich aus der wässerigen Lösung sehr schöne, gelbe Krystalle aus, welche bei $195^{\circ}$ unter Zersetzung schmolzen.

Die alkoholischen Mutterlangen $B$ wurden zur späferen Untersuchung anfbewahrt.

Die auf die beschriebene Art gewonnene Verbindung ist eine Säure, welche kohlensaure Salze mit Leichtigkeit zersetzt, eine gelbliche Farbe hat, leicht löslich in Alkohol, schwieriger in Äther, fast unlöslich in Chloroform ist. Die wässerige Lösung 
gibt mit Eisenchlorid eine intensive grasgrüne Färbung, die auf Zusatz von Soda dunkelroth wird. Bromwasser bewirkt in der Lösung zuerst eine duukelbraune Färbung, dann einen braunen Niederschlag. Die wässerige Lösnng reducirt eine Lösung von Silbernitrat. Essigsaures Blei gibt einen citronengelben Niedersehlag. Alle diese aufgezählten Eigenschaften hat die Substanz mit der Kaffeesäure gemein.

Die Substanz der Elementaranalyse unterworfen, lieferte aber zu viel Kohlenstoff, so dass die Vermuthung nahe lag, dass dieselbe trotz des constanten Schmelzpunktes noch nicht ganz rein sei. Auch gab die Substanz eine kleine Methylzabl.

Die weitere Reinigung der Substanz wurde in der Weise vorgenommen, dass sie mehreremale mit Chloroform ausgekocht wurde. Das Chloroform löste eine geringe Menge eines Körpers $C$, welcher später beschrieben werden wird. Am vortheilhaftesten schien es, die Substanz $C$ im Extractionsapparate mit Chloroform zu lösen. Der so gereinigte Körper wurde neuerdings mit Wasser umkrystallisirt und gab dann keine Methylzabl mehr. Der Schmelzpunkt ist bei $195^{\circ}$.

Die Analyse der bei $120^{\circ}$ getrockneten Substanz ergab:

I. $0 \cdot 3369 g$ Substanz gaben $0 \cdot 7481 g$ Kohlensäure und $0 \cdot 1356 g$ Wasser.

II. $0 \cdot 359 g$ Substanz gaben $0 \cdot 795 g$ Kohlensäure und $0 \cdot 146 g$ Wasser.

III. $0 \cdot 3788 g$ Substanz gaben $0 \cdot 8342 g$ Kohlensäure und $0 \cdot 1454 g$ Wasser.

In 100 Theilen:

$$
\begin{aligned}
& \text { I. II. III. } \mathrm{C}_{9} \mathrm{H}_{8} \mathrm{O}_{4}
\end{aligned}
$$

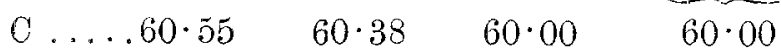

$$
\begin{aligned}
& \text { Н....4.47 } 4.51 \quad 4 \cdot 26 \quad 4 \cdot 44 \text {. }
\end{aligned}
$$

Die Resultate der Verbrennungen stimmen gut zu der Formel der Kaffeesäure. Die frtuher angefülurten Eigenschaften der aus dem Harz dargestellten krystallisirten Säure, sowie die Eigenschaften der noch später folgenden Derivate derselben liefern 
den Beweis, dass die dargestellte Substanz nichts anderes als Kaffeesäure

$$
\mathrm{C}_{6} \mathrm{H}_{3} \leq \begin{aligned}
& \mathrm{CH}=\mathrm{CH}-\mathrm{COOH}(1) \\
& \mathrm{OH}(4)
\end{aligned}
$$

ist.

Sie wurde znerst von Hlasiwet ${ }^{1}$ ans der Kaffeegerbsäure, später dann von Tiemann und $\mathrm{Nagai}^{2}$ aus dem Protocatechualdehyd dargestellt. $\mathrm{H}_{0}$ fman ${ }^{3}$ fand die Kaffeesäure im Schierling.

Es findet sich in der Literatur keine Angabe, dass Kaffeesänre in einem Harze gefunden worden wäre.

Die von mir ans dem Überwallungsharz dargestellte Kaffeesäure wurde mit der in der Präparatensammlung des Laboratoriums vorhandenen, von Hlasiwetz dargestellten verglichen und reigte sich mit dieser in allen Punkten identisch. Nur in Bezug auf den Wassergehalt ergab sich eine kleine Differenz. Hlasiwetz gibt an, dass die Kaffeesäure ein halbes Molekuil Wasser enthalte, während ich bei der aus dem Harze dargestellten Säure entweder gar kein Wasser oder so viel fand, dass die Menge so ziemlich auf ein Molekiil stimmte.

Es zeigte sich beim Umkrystallisiren ans Wasser, dass aus concentrirten heissen Lösungen die Säure wasserfrei krystallisirt, alls verdünnten wasserbaltig.

Aus verdünnten Lösungen erhaltene Krystalle gaben bei der Wasserbestimmung:

I. $0.4142 \mathrm{~g}$ Substanz verloren bei $120^{\circ} 0.0354 \mathrm{~g}$ Wasser. II. $0 \cdot 3928 g$ Substanz verloren bei $120^{\circ} 0 \cdot 0338 q$ Wasser.

In 100 Theilen:

$$
\text { I. II. } \quad \mathrm{C}_{9} \mathrm{H}_{8} \mathrm{O}_{4}+\mathrm{H}_{2} \mathrm{O}
$$

$$
\text { Wasser ....8 } 54 \quad 8 \cdot 60 \quad 0.09 \text {. }
$$

1 Annalen der Chem. und Pharm., 142, 221.

2 Berliner Berichte, 11, 657.

3 Ebenda, 17, 1922. 
Es wurden behufs weiterer Feststellung der Identität beider Verbindungen noch zwei Derivate derselben dargestellt.

\section{Darstellung des Acetylproductes.}

Zur Darstellung desselben wurde die Substanz mit der vierfachen Menge von Essigsäureanhydrid längere Zeit am Rückfiasskühler gekocht und nach beendeter Einwirkung" in Wasser" gegossen. Es löste sich alles in demselben anf und erstarrte nach kurzer Zeit zu einem krystallinisehen Brei, der abgesaugt, mit Wasser ansgekocht und dann mit verdünntem Alkohol umkrystallisirt wurde.

Man erhält so das Acetylproduct in feinen, weissen Nadeln, die bei $189^{\circ}$ schmelzen.

Die Verbindung ist in Wasser sehr schwer löslich, leicht in Alkohol und Äther. Eisenchlorid gibt mit der Lösung dieser Verbindung keine griine Färbung mehr.

Die Analyse der bei $120^{\circ}$ getrockneten Substanz ergab nachstehende Resultate:

I. $0 \cdot 327 g$ Substanz gaben $0 \cdot 713 g$ Kohlensäure und $0 \cdot 1336 g$ Wasser.

II. $0.3926 g$ Substanz gaben $0.8564 g$ Kohlensäure und $0 \cdot 1615 g$ Wasser.

III. $0.2756 g$ Substanz gaben $0 \cdot 6016 g$ Kohlensäure und $0 \cdot 1129 g$ Wasser.

In 100 Theilen:

\begin{tabular}{crcc} 
I. & II. & \multicolumn{1}{c}{ III. } & $\mathrm{C}_{9} \mathrm{H}_{6}\left(\mathrm{C}_{2} \mathrm{H}_{3} \mathrm{O}_{2} \mathrm{O}_{4}\right.$ \\
$\mathrm{C} \ldots \ldots .59 \cdot 44$ & $59 \cdot 49$ & $59 \cdot 50$ & $59 \cdot 09$ \\
$\mathrm{H} \ldots \ldots .4 .52$ & $4 \cdot 55$ & 4.54 & 4.54.
\end{tabular}

Tiemann und Nagai ${ }^{1}$ stellten znerst dic Diacetylkaffee-

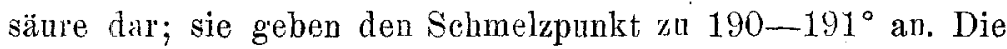
Eigenschaften der von mir dargestellten Diacetkaffeesäure stimmen vollständig mit den Angaben der oben genannten Forscher.

1 Berliner Berichte, 11, 6506 . 


\section{Einwirkung von Natriumamalgam.}

$5 g$ der Säure wurden in einein Kolben mit einer angemessenen Menge von Wasser und Natriumamalgam am Rỉckflusskuihler durch eine Stunde gekocht. Die Lösung wurde sodann in der Weise neutralisirt, dass die Schwefelsäure durch das Kühlrohr des Kühlers in die Lösung gegossen wurde, da die alkalische Lösung der Hydrokaffeesäure für Luftzutritt ausserordentlich empfindlich ist. Die so abgesättigte Flissigkeit wurde nun mehreremale mit Äther ausgeschüttelt, auf welche Weise man einen syrupösen Rüickstand erhielt. Durch öfteres Umkrystallisiren aus Wasser wurden schön ausgebildete Krystalle erhalten, welche sich sebr leicht in Wasser lösten.

Die Lösung wird durch Eisenchlorid intensiv grün gefärbt; die alkalische Lösung des Hydroproductes ist schwach röthlich gefärbt. Die Säure schmilzt bei $139^{\circ}$.

Dieses Hydroproduct zeigt sich mit der in der Sammlung: des Laboratoriums vorhandenen, von Hla siw etz ${ }^{1}$ dargestellten Hydrokaffeesäure vollständig identisch. Der Schmelzpunkt der Hydrokaffeesäure von Hlasiw etz ist zwar nicht in der Literatur angegeben, er wurde von mir bestimmt und ist ebenfalls bei $139^{\circ}$.

Trotz dieser Übereinstimmung sind die Resultate der Analyse bezüglich des Kohlenstoffes zu hohe.

Die Analysen der bei $100^{\circ}$ getrockneten Substanz lieferten nachstehende Werthe:

I. $0.2417 g$ Substauz gaben $0.5364 g$ Kohlensäure und $0.1218 g$ Wasser.

II. $0 \cdot 2884 g$ Substan\% gaben $0 \cdot 6399 g$ Kohlensäure und $0 \cdot 143 g$ Wasser.

In 100 Theilen:

$$
\begin{array}{rrr}
\text { C...60.40 } & 60.50 & 59 \cdot 30 \\
\mathrm{H} \ldots .5 .58 & 5.51 & 5.50 .
\end{array}
$$

Herr Dr. J. Hocka uf, Assistent am mineralogischen Museum des Herrn Prof. Schrauf an der hiesigen Universität, hatte die Freundlichkeit, die Krystalle der von mir dargestellten Hydro-

1 Annalen der Chem. und Pharm., 142, 354. 
kaffeesäure zu messen und den krystallographischen Vergleich mit der Hydrokaffeesäure von Hlasiwetz durchzufuhren. Ich erlaube mir, an dieser Stelle Herrn Dr. Hockauf für seine freundliche Bemühung meinen verbindlichsten Dank auszusprechen.

Derselbe theilt mir Folgendes mit:

"Sechsseitige Krystalle nach $c=(001)$ tafelförmig entwickelt. Beobachtete Formen $c=(001), a=(100)$, $p=(111)$. Die Basisfläche muldenförmig vertieft, die Pyramidenflächen facettirt, Kanten verkrümmt. Gute Signale nur an

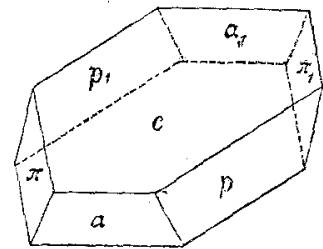

Fig. 1. einer Pinakoid- und an einzelnen Pyramidenflächen. Selbst zusammengehörige Flächen sind nicht vollkommen parallel. Ein $4 \mathrm{~mm}$ langer, $3 \mathrm{~mm}$ breiter Krystall, welcher der Messung unterzogen wurde, ergab nachstehende Werthe:

\begin{tabular}{|c|c|c|c|}
\hline \multicolumn{2}{|c|}{ Beobachtet } & \multicolumn{2}{|c|}{ Monoklin gerechnet } \\
\hline$a: c=(100):(001)=72^{\circ}$ & $22^{\prime}$ & $* 72^{\circ}$ & \\
\hline$a_{1}: c_{1}=(100):(00 \overline{1})=71$ & 38 & 72 & \\
\hline$c: a_{1}=(001):(\overline{1} 00)=106$ & 45 & 108 & \\
\hline$c_{1}: a=(001):(100)=109$ & 14 & 108 & \\
\hline$a: p=(100):(111)=63$ & 20 & $* 62$ & $50^{\prime}$ \\
\hline$\pi: a=(1 \overline{1} 1):(100)=62$ & 37 & 62 & 50 \\
\hline$\pi_{1}: a_{1}=(\overline{1} 1 \overline{1}):(\overline{1} 00)=62$ & 15 & 62 & $50^{\circ}$ \\
\hline$p_{1}: a=(111):(100)=63$ & 10 & 62 & 50 \\
\hline$p: a_{1}=(111):(100)=115$ & 32 & 117 & 10 \\
\hline$a_{1}: \pi=(\overline{1} 00):(1 \overline{1} 1)=118$ & 1 & 117 & 10 \\
\hline$a: \pi_{1}=(100):\langle\overline{1} 1 \overline{1}\rangle=117$ & 13 & 117 & 10 \\
\hline$p_{1}: a==(111):(100)=117$ & 39 & 117 & 10 \\
\hline$p: \pi=(111):(111)=105$ & 15 & 104 & 59 \\
\hline$p_{1}: \pi_{1}=(\overline{1} 1 \overline{1}):(\overline{1} 1 \overline{1})=104$ & 48 & 104 & 59 \\
\hline$\pi: p_{1}=(1 \overline{1} 1):(111)=75$ & 57 & 75 & 1 \\
\hline$\pi_{1}: p=(\overline{1} 1 \overline{1}):(111)=74$ & & 75 & 1 \\
\hline$p: c=(111):(001)=58$ & 41 & $* 58$ & 22 \\
\hline$p_{1}: c_{1}=(\overline{1} \overline{1}):(00 \overline{1})=56$ & 36 & 58 & 22 \\
\hline$\pi: c=(1 \overline{1} 1):(001)=58$ & 47 & 58 & 22 \\
\hline$\pi_{1}: c_{1}=(11 \overline{1}):(00 \overline{1})=59$ & 25 & 58 & 22 \\
\hline$c: p_{1}=(001):(1 \overline{1})=121$ & 30 & 121 & 38 \\
\hline$c_{1}: p=(00 \overline{1}):(111)=123$ & 11 & 121 & 38 \\
\hline$c: \pi_{1}=(001):(\overline{1} 1 \overline{1})=120$ & 55 & 121 & 38 \\
\hline$c_{1}: \pi=(00 \overline{1}):(1 \overline{1})=120$ & 52 & 121 & 38 \\
\hline Hoft Nr. 8 . & & & 33 \\
\hline
\end{tabular}

Chemie-Hoft Nr. 8. 
Die Winkel $72^{\circ}, 62^{\circ} 50^{\prime}, 58^{\circ} 22^{\prime}$ sind Mittelwerthe. Auf Grund derselben wurde das monosymmetrische Axenverhältniss $a: b: c=2 \cdot 5604: 1: 1 \cdot 9676, n=108^{\circ}$ gerechnet.

Nach obigen Messungen sind die Krystalle asymmetrisch, mit grosser Annäberung an Monosymmetrie. Da jedoch $b(010)$ fehlte, konnte ein genaues asymmetrisches Axenverbältniss nicht gerechnet werden.

Krystalle lichtbräunlich, durchsichtig; starke Doppelbrechung. Optisch zweiaxig. Auf (001) tritt ein aus; sie liegt seitlich der scheinbaren Symmetrieebene nabe der Kante $a_{1} / c$. Die Auslöschungsrichtung bildet auf 001 mit der Kante $a / c$ einen Winkel von circa $6 \frac{1}{2}$.

Die Hydrokaffeesäure $\mathrm{Hlasiwetz}$ ist krystallographisch und optisch iibereinstimmend mit der Hydrokaffeesäure Bamberger:"

\section{Aufarbeitung von $B$.}

Es wurde bereits früher erwähnt, dass die Kaffeesäure bei der Analyse anfangs immer zu hohe Zahlen für den Kohlenstoff lieferte und der Grund hiefür darin lag, dass der Säure noch ein zweiter, dureh Chloroform extrahirkarer Körper $C$ beigemengt war. Die grösste Menge dieses Körpers befand sich aber nun in den alkoholischen Mutterlaugen, welche beim Absaugen der Kaffeesäure erhalten wurden. Aus denselben krystallisirte sebr bald ein Körper, der von der Mutterlauge durch Aufstreichen auf Thonplatten getrennt wurde. Die trockene Masse wurde in Chloroform gelöst; nach dem Einengen der Lösung schieden sich schöne weisse Krystalle ans, die abfiltrirt und mit heissem Wasser umkrystallisirt wurden.

Man erhielt so aus der wässerigen Lösung sehr schöne, lange, farblose, irisirende Nadeln, welche sich leicht in Alkohol and in Äther lösten. In kaltem Wasser sind sie fast unlöslich. Doch löst sich die Verbindung mit gelber Farbe in Alkalien. Die wässerige Lösung dieser Verbindung gibt mit Bleizuckerlösung. eine reichliche gelbe Fällung und mit Eisenchlorid einen dunkelgelbbrannen Niederschlag. Die Verbindung schmilzt bei $169^{\circ}$.

Alle diese Eigenschaften hat die Verbindung mit der Ferulas äure 


$$
\mathrm{C}_{6} \mathrm{H}_{3} \frac{\mathrm{CH}=\mathrm{CH}-\mathrm{COOH}}{\mathrm{OH}}
$$

gemein. Die Analyse der bei $100^{\circ}$ getrockneten Substanz ergab: $0.3022 \mathrm{~g}$ Substanz gaben $0 \cdot 6794 \mathrm{~g}$ Kohlensäure und $0.1337 \mathrm{~g}$ Wasser.

In 100 Theilen:

$$
\begin{array}{lr}
\mathrm{C} \ldots \ldots 61 \cdot 36 & \mathrm{C}_{10} \mathrm{~B}_{10} \mathrm{O}_{4} \\
\mathrm{H} \ldots \ldots .4 .92 & 61 \cdot 85 \\
5 \cdot 15 .
\end{array}
$$

Ausserdem wurde noch die Methylzahl der Säure bestimmt.

I. $0.4227 g$ bei $100^{\circ}$ getrockneter Substanz gaben $0.4942 g$ Jodsilber.

II. $0.3736 g$ bei $100^{\circ}$ getrockneter Substanz gaben $0 \cdot 440 g$ Jodsilber.

In 1000 Theilen:

$$
\begin{array}{ccc}
\text { I. } & \text { II. } & \mathrm{C}_{10} \mathrm{H}_{10} \mathrm{O}_{4} \\
74 \cdot 6 & 75 \cdot 2 & 77 \cdot 4 .
\end{array}
$$

Es wurde ferner auch die Bestimmung der Basicität der bei $100^{\circ}$ getrockneten Säure nach der Methode von $\mathrm{Fuchs}^{1}$ vorgenommen, welche nachfolgende Resultate ergab:

I. $0 \cdot 0966 \mathrm{~g}$ Substanz entwickelten bei einem Druck $B=73+\mathrm{mm}$ und einer Temperatur $T=15^{\circ}$ an Schwefelwasserstoff $11.8 \mathrm{~cm}^{3}$.

II. $0.0896 \mathrm{~g}$ Substanz entwickelten bei einem Druck $B=752 \mathrm{~mm}$ and einer Temperatur $T=17.5^{\circ}$ an Schwefelwasserstoff $11 \cdot 1 \mathrm{~cm}^{2}$.

In Procenten ersetzbaren Wasserstoffs

$\overbrace{\text { I. }}^{\text {II. }} \begin{gathered}0.591 \\ 0.505\end{gathered} \frac{\mathrm{C}_{10} \mathrm{H}_{10} \mathrm{O}_{4}}{0.51 .}$

1 Monatshefte für Chemie, IX, 1132. 
Die Ferulasäure wurde zuerst von Hasiwetz und Barth ${ }^{1}$ in der Asa foetida aufgefunden, dann von Tiemann und $\mathrm{Nagai}^{2}$ durch Kochen von Acetylferulasäure mit Kalilauge dargestellt.

Die von mir gewonnene Ferulasäure zeigte mit der in der Präparatensammlung des Laboratoriums yorhandenen, von Hlasiwetz und Barth dargesteliten, vollständige Übereinstimmung. Anch der Schmelzpunkt der Säure aus der Asa foetida beträgt $169^{\circ}$.

Man kann die Ferulasäure auch nach der Methode, welche Hlasiwetz und Barth bei der Darstellung aus der Asa foetida angeben, zu ihrer Gewinnung aus dem Überwallungshar\%e anwenden.

Es wird das Harz in Alkohol gelöst, die Lösung mit alkoholischer Bleizuckerlösung gefällt, der Niederschlag abfiltrirt, ausgepresst und mit verdinnter Schwefelsäure zersetzt. Man erhält so ein Gemenge von Kaffeesäure und Ferulasänue, welche dann durch Chloroform getrennt werden müssen.

Die Ausbente an Kaffeesäure und Ferulasäure ist aber viel besser, wenn man das Verfahren, das ich früher beschrieben habe, anwendet, nämlich das Auskochen mit Wasser.

Die Ausbente an Kaffeesäure und Ferulasäure beträgt fur die erstere circa $4 \%$, für letztere circa $1 \%$ des angewandten Harzes. Es muss hiebei bemerkt werden, dass selbst durch oftmaliges Auskochen des Harzes mit Wasser dasselbe nicht an beiden obgenannten Säuren ersehöpft werden konnte. Die Säurezahl des viermal mit Wasser ausgekochten Harzes ist 58, die Methylzahl betrïgt $52 \cdot 39$, ist also fast ganz dieselbe, wie die des unausgekochten Harzes.

Die wässerigen Lösungen der letzten Harzabkochungen geben mit Bleizuckerlösung noch immer sehr reichliche gelbe Fällungen. Schüttelt man die letzten wässerigen Lösungen mit Äther ans und destillirt denselben ab, so bekommt man Syrupe, die sehr schwer krystallisiren.

Es ist wohl sicher anzanehmen, dass die Kaffeesäure und Ferulasäure präformirte Bestandtheile des Überwallungsharzes

1 Annalen der Chem. und Pharm., 138, 64.

2 Berliner Berichte, 9, 416 und 11, 649 . 
bilden, da sie nacb einem so einfachen Verfahren gewounen werden können. Es ist noch zu erwähnen, dass sich beim Auskochen des Harzes ein penetranter Geruch nach Buttersäure bemerkbar machte.

Darstellung des Vanillins aus dem Überwallungsharz.

Es wurde bereits früher angedeutet, dass das Harz eine sehr starke Phloroglucinreaction gibt. Um num das Vanillin zu isoliren, wurden die alkobolischen Mutterlaugen $\boldsymbol{B}$, aus denen die Ferulasäure entfernt wurde, eingedampft, der Rückstand in Äther gelöst und die ätherische Lösung mit Natritumbisulfit ausgeschüttelt. Die weitere Aufarbeitung geschah nach der Methode, welche Tiemann und Haarmann ${ }^{1}$ zur quantitativen Bestimmung des Vanillins in der Vanille angeben. Schliesslich warde das Vanillin durch Umikrystallisiren aus Petroleumäther gereinigt.

Die so gewonnene Substanz zeigte alle Eigenschaften des Vanillins

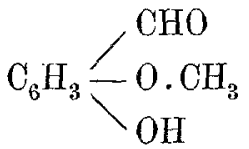

Die Methoxylirung desselben ergab nachstehende Methylzahl: $0 \cdot 0764 g$ Substanz gaben $0 \cdot 1156 g$ Jodsilber.

In 1000 Theilen:

$$
96 \cdot 5 \quad \frac{\mathrm{C}_{8} \mathrm{H}_{8} 0_{3}}{98 \cdot 6 .}
$$

Die Quantität des gewonnenen Vanillins war eine gering'e. Das Vanillin wurde von Jannasch und Rump ${ }^{2}$ in dem SiamBenzoëharz und von E. Schmidt ${ }^{3}$ in der Asa foetida aufgefunden.

Abietinsäure aus dem Harze darzustellen, ist mir bis jetzt noch nicht gelungen, womit ich natïrlich nicht behaupten will,

1 Berliner Berichte, 8, 1115 .

2 Elyenda, 11, 1635 .

3 Arch. Pharm. [3], 24, 534-535 und Berliner Berichte, 19, $705 c$. 
dass keine vorhanden sei, aber nach den bekannten Nethoden zur Isolirung derselben konnte ich keine finden.

Einwirkung von schmelrendem Kali auf das ausgekochte Überwallungshar'z.

$500 \mathrm{~g}$ ausgekochtes Harz wurden mit $2 \mathrm{~kg}$ Kali in einer eisernen Schale durch circa eine Stunde geschmolzen. Anfangs schmilzt das Harz als zäher Klumpen auf dem geschmolzenen Ätzkalium, nach etwa zehn Minuten mischte es sich mit demselben. Die Masse wird dann homogen, beginnt selr stark zu schäumen, auch ist eine lebhafte Gasentwicklung bemerkbar.

Das Schmelzen wurde unterbrochen, als die Masse einzusinken begann. Dieselbe wurde in Wasser gelöst und mit einem Übersehuss von verdiunter Schwefelsäure versetzt. Es schied sich eine ziemlich bedeutende Menge einer schwarzen Masse aus, von welcher die Fliissigkeit abfiltrirt wurde. Die abgesättigte, von Harz und ausgeschiedenem schwefelsauren Kalium befreite Flüssigkeit wurde dreimal mit Äther ausgeschüttelt; nach dem Abdestilliren desselben hinterblieb im Kolben eine dicke bräunliche Masse, welche in Wasser gelöst wurde. Nach ein paar Stunden erstarrte die wässerige Lösung zu einer krystallinischen Masse, welche stark nach Essigsäure roch. Dieselbe wurde nun in heissem Wasser gelöst und die Lösung mit kohlensaurem Baryt neutralisirt. Es wurde abfiltrirt, das Filtrat dreimal mit Äther ausgeschüttelt, nm die etwa vorhandenen phenolartigen Körper zu entfernen. Nach dem Verjagen des Äthers blieb im Destillationskolben eine syrupartige Masse, welche nach längerer Zeit krystallinisch erstarrte. Die Eigenschaften des Körpers wiesen auf Brenzcatechin, so entstand mit Eisenchlorid ein griunlich-schwarzer flockiger Niederschlag. Das Brenzcatechin wurde nicht weiter gereinigt, da es ja höchst wahrscheinlich aus der bei der Kalischmelze gebildeten Protocatechusäure entstanden war und daher kein weiteres Interesse bot.

Die vom Brenzeatechin befreite Flüssigkeit wurde mit verdünnter Schwefelsäure versetzt, vom schwefelsauren Baryt abfiltrirt und das Filtrat mehreremale mit Äther ausgeschüttelt. Beim Ver-

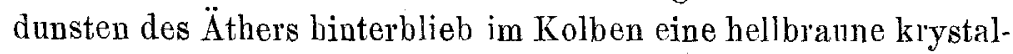
linische Masse, welche beim öfteren Umkrystallisiren aus Wasser 
schöne glänzende Nadeln gab, die bei $194^{\circ}$ schmolzen. Eisenchlorid färbt die Lösung des Körpers gr'tỉn.

Die Analyse der bei $120^{\circ}$ getrockneten Substanz ergab folgende Resultate:

$0.4711 \mathrm{~g}$ Substanz gaben $0.9458 \mathrm{~g}$ Kohlensäure und $0.1655 \mathrm{~g}$ Wasser.

In 100 Theilen:

$$
\begin{array}{lr} 
& \frac{\mathrm{C}_{7} \mathrm{H}_{6} \mathrm{O}_{4}}{\mathrm{C} \ldots \ldots 54 \cdot 74} \\
\mathrm{H} \ldots \ldots .5 \cdot 90 & 54 \cdot 54 \\
& 3 \cdot 89 .
\end{array}
$$

Die lufttrockene Verbindung enthält ein Molekiil Krystallwasser, wie folgende Bestimmung zeigt:

$0.5267 \mathrm{~g}$ Substanz verloren bei $120^{\circ}$ getrocknet $0.0556 \mathrm{~g}$ Wasser.

In 100 Theilen:

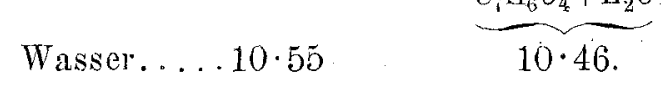

Die bei der Kalischmelze erhaltene Substanz ist also Protocatechusäure. Aus $500 \mathrm{~g}$ wurden etwa $20 \mathrm{~g}$ Sänre erhalten.

Die Protocatechusäure entsteht wohl in der Kalischmelze ans der Ferulasänre, eine Thatsache, die bereits von Hlasiwetz und Barth ' angegeben wurde und durch folgende Gleichung zum Ausdrucke kommt:

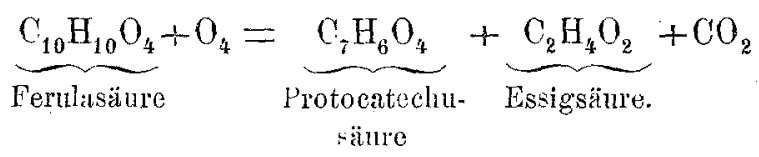

Das Überwallungsharz der Fichte.

Die naturhistorische Charakterisirung des Harzes von Herrn Dr. J. Krasser wurde im Eingange der Arbeit gegeben.

Herrn L. Elamann, der die Güte hatte, mir das Harz in Velden zu beschaffen, sage ich meinen besten Dank.

1. Annalen der Chem. und Pharm., 138, 68. 
Der Schmelzpunkt des Harzes ist bei circa $100^{\circ}$. Die Säure-, Jod- und Methylzahlen wurden wie beim Überwallungsharz der Schwarzföhre ermittelt und haben nachfolgende Werthe ergeben:

\begin{tabular}{|c|c|c|}
\hline SäurezahI & Jodzahl & Methylzab! \\
\hline $127 \cdot 7$ & $61 \cdot 2$ & $34 \cdot 9$ \\
\hline 125 & - & 33 \\
\hline
\end{tabular}

Das Harz wurde ganz analog der Methode, wie bei dem Uberwallungsharz der Föbre beschrieben wurde, aufgearbeitet. Man erhält so beim Abdestilliren des Äthers einen sehr schönen, weissen, krystallinisehen Ritekstand, der zuerst ans Alkohol umkrystallisirt, später durch Kochen mit Chloroform von anderen Veranreinigungen befreit wurde. Die Substanz wurde schliesslich in heissem Wasser gelöst, aus dem sie in schönen weissen Nadeln krystallisirte.

Die zuerst anschiessenden Krystalle sind sichelartig gekrümmt und garbenartig verwachsen. Kaltes Wasser löst sehr

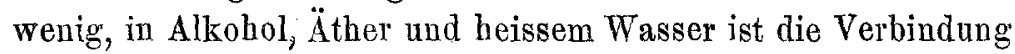
leicht löslich.

Der Schmelzpunkt wurde bei $204^{\circ}$ gefunden. Eisenchlorid gibt mit der alkoholisehen Lösung eine dunkelbraune Färbung. Die Substanz reagirt stark sauer, reducirt nicht Silbernitratlösung.

Die bei $120^{\circ}$ getrocknete Substanz der Elementenanalyse unterzogen, lieferte nachstehende Resultate:

I. $0 \cdot 2621 g$ Substanz gaben $0 \cdot 6304 g$ Kohlensäure und $0 \cdot 114 g$ Wasser.

II. $0 \cdot 3002 g$ Substanz gaben $0 \cdot 722 g$ Kohlensäure und $0 \cdot 127 g$ Wasser.

III. $0 \cdot 284 g$ Substanz gaben $0 \cdot 6842 g$ Kohlensäure und $0 \cdot 1236 g$ Wasser.

In 100 Theilen:
I.
II.
III.
$\mathrm{C}_{9} \mathrm{H}_{8} \mathrm{O}_{3}$
C....65.58
$65 \cdot 60$
$65 \cdot 70$
$65 \cdot 85$
H.... 4.83
$4 \cdot 69$
$4 \cdot 83$
$4 \cdot 87$. 
Die Säure hat alle Eigenschaften der schon von Rochleder ${ }^{1}$ durch Behandlung von Aloë mit verdünnter Natronlauge hergestellten Paracumarsäure

$$
\mathrm{C}_{6} \mathrm{H}_{4}<\begin{aligned}
& \mathrm{CH}=\mathrm{CH}-\mathrm{COOH} \\
& \mathrm{OH}
\end{aligned}
$$

Hlasiwetz ${ }^{2}$ hat dann gezeigt, dass sich dieselbe auch beim Behandeln von Aloë mit verdinnter Schwefelsätre bildet. Später wurde diese Säure von Tiemann und Herzfeld ${ }^{3}$ aus Paraoxybenzaldehyd, dann von Jobst und Hesse ${ }^{4}$ aus der Cotorinde dargestellt. Gabriel ${ }^{5}$ gewann die Verbindung aus Paraamidozimmtsäure durch Diazotirung und Will ${ }^{6}$ stellte sie ans dem Naringenin dar und nannte sie Naringeninsäure.

Die von mir dargestellte Säure krystallisirt aus kaltem Wasser mit einem Molekuil Wasser, aus heissem fast wasserfrei. Erstere Thatsache wurde zuerst von Rochleder constatirt. Auch Will findet, dass die Naringeninsäure, welche identisch mit der Paracumarsäure ist, aus kalter wässeriger Lösung mit einem Volekül Wasser krystallisirt.

Die Wasserbestimmung, welche mit der aus heissem Wasser erhaltenen Paracumarsăture vorgenommen wurde, gab nachstehendes Resultat:

$0 \cdot 2642 g$ Substanz gaben $0 \cdot 0028 g$ Wasser.

In 100 Theilen:

$$
\text { Wasser ......... } 05 \% \text {. }
$$

Die Wasserbestimmung der aus kaltem Wasser erhaltenen Säure ergab:

I. $0 \cdot 291 \mathrm{~g}$ Substanz gaben $0.0289 \mathrm{~g}$ Wasser.

II. $0.397 \mathrm{~g}$ Substanz gaben $0.0382 \mathrm{~g}$ Wasser. III. $0 \cdot 3334 g$ Substanz gaben $0.033 g$ Wasser.

1 Annalen der them. und Pharm., 136, 35.

2 Ebenda, 136, 131.

3 Berliner Berichte, 10, 65 .

4 Ebenda, 10, 249.

5 Ebenda, 15, 2301.

6 Ebenda, 20, 299. 
In 100 Theilen:

$\begin{array}{cccc}\text { I. } & \text { II. } & \text { III. } & \frac{\mathrm{C}_{3} \mathrm{H}_{8} \mathrm{O}_{3}+\mathrm{H}_{2} \mathrm{O}}{9 \cdot 89 .}\end{array}$

Nach der Methode von F u chs wurde auch hier die Basicität der Säure bestimmt und nachstehendes Resultat erhalten:

I. $0.0444 \mathrm{~g}$ der bei $120^{\circ}$ getrockneten Substanz gaben bei einem Druck $B=746 \mathrm{~mm}$ und einer Temperatur $T=21 \cdot 5^{\circ}$ an Schwefelwasserstoff $6 \cdot 7 \mathbf{c m}^{3}$.

II. $0 \cdot 0466 \mathrm{~g}$ Substan\% gaben bei einem Druck $B=749 \mathrm{~mm}$ und einer Temperatar $T=22^{\circ}$ an Schwefelwasserstoff $7 \mathrm{~cm}^{3}$.

In Procenten ersetz-

baren Wasserstoffs

$\frac{\mathrm{I}_{0.599}}{0.597} \quad \frac{\mathrm{C}_{9} \mathrm{H}_{8} \mathrm{O}_{3}}{0.609 .}$

Die Ausbeute an Paracnmarsäure betrug circa $2 \%$ des angewandten Harzes.

Die von mir erhaltene Paracumarsäure zeigte sich im Vergleiche zu der von Hlasiwetz dargestellten, in der Sammlung des Laboratoriums vorhandenen, als vollständig identisch.

Zur weiteren Identificirung wurde noch das Hydroproduct der Paracumarsäure dargestellt.

Einwirkung von Natriumamalgam.

$5 g$ in Wasser suspendirter Säure wurden mit der angemessenen Menge von Natriumamalgam durch $3 / 4$ Stunden gekocht. Die mit verdünnter Schwefelsäure neutralisirte Flüssigkeit wurde dreimal mit $\ddot{A}$ ther ausgeschüttelt. Nach dem Abdestilliren des Äthers, Auflösen des syrupartigen Rückstandes in Wasser, krystallisirte das Hydroproduct sehr bald in schönen, ziemlich grossen Krystallen. Das Hydroproduct löst sich sebr leicht in Wasser und Äther und schmilzt bei $128^{\circ}$.

Die Analyse der bei $100^{\circ}$ getrockneten Verbindung gab nachstehendes Resultat:

$0.2622 g$ Substanz gaben $0.6245 g$ Koblensäure und $0.1405 g$ Wasser. 
In 100 Theilen:

$$
\begin{array}{lr}
\text { C. . . } & \mathrm{C}_{9} \mathrm{H}_{1}, \mathrm{O}_{3} \\
\mathrm{H} \ldots \ldots .95 & 65.06 \\
6.95 \cdot 02 .
\end{array}
$$

Die Säure hat alle Eigenschaften der zuerst von Hlasiwetz ${ }^{2}$ dargestellten $\mathrm{Hy}$ dropar a cumarsäur e.Glaser und Bucbanan ${ }^{2}$ erhielten sie später aus der Paranitrozimmtsäure. Baumann ${ }^{3}$ fand sie im menschlichen Harn. Die Hydronaringeninsäure von Will * ist wohl identiseh mit der Hydroparacnmarsänre.

\section{Darstellung des Vanillins.}

Auch dieses Harz gibt eine ziemlich starke Phloroglueinreaction. Das Vanillin wurde aus den alkoholisehen Mutterlaugen, von der Darstellung der Paracumarsäure herruhrend, ganz analog, wie schon früher beschrieben, isolirt. Die Menge desselben war gering.

Die Säure- und Methylzahl des ausgekochten Harzes wurden bestimmt und Nachstehendes gefunden:

$\underbrace{\text { Sänrezahl }}_{99} \quad \underbrace{\text { Methylzahl }}_{34 \cdot 8 .}$

Ferulasäure in diesem Harze nachzuweisen, ist mir bis jetzt noch nicht gelungen, ebensowenig Abietinsäure. Ich erhielt wohl aus den alkoholischen Mutterlaugen, von der Darstellung der Paracumarsäure herrihiend, einen krystallisirten Körper, der einige Eigenschaften der Ferulasäure zeigte, allein die Menge desselben war so gering; dass an eine näbere Untersuchung nicht gedacht werden konnte.

Einwirkung ron sehmelzendem Kali auf das ausgekochte Harz.

$160 \mathrm{~g}$ des Harzes wurden mit der zebnfachen Menge ron Kali durch $3 / 4$ Stunden in einer eisernen Pfanne geschmolzen.

1 Annalen der Chem. und Pharm, 142, 358.

2 Zeitschrift für Chemie, 12, 193.

3 Berlint $\mathbf{r}$ Berichte, 13, 1881.

4 Ebenda, 20, 299. 
Es ist nothwendig, einen bedeutenden Überschuss an Kali zu nehmen, da mit einer geringen Menge die ganze Masse fest wird. Es dauert sehr lange, bis sich das geschmolzene Harz und Kali mischen. Als die Masse ruhig floss und eine dunkelbraune Färbung hatte, wurde mit dem Schmelzen anfgehört. Dieselbe wurde in Wasser gelöst und ganz analog, wie dies schon bei dem ersten Harze beschrieben wurde, aufgearbeitet.

Von phenolartigen Körpern war wieder Brenzeatechin zu finden. Beim Ausschtitteln der von dem genannten Körper befreiten Flüsigkeit wurde ein Gemenge von zwei Säuren erhalten. Die wässerige Lösung derselben gab mit Eisenchlorid eine intensive gräne Färbung, mit Bromwasser eine reichliche gelbe Fällung. Es war also anzunehmen, dass ein Gemisch von Protocatechusäure und Paraoxybenzoësäure vorliege.

Hlasiwetz and Barth ${ }^{1}$ fanden ebenfalls beim Schmelzen von Benzoëharz mit Kali ein Gemenge oben genannter Säuren, die sie aber durch Krystallisation nicht trennen konnten. Das Gemenge, das mir vorlag; konnte ganz gut durch Krystallisation getrennt werden.

Ich erhielt auf diese Art kleine Prismen, die sich leicht in heissem Wasser lösten und einen Schmelzpunkt von $210^{\circ}$ besassen:

Die Analyse der bei $120^{\circ}$ getrockneten Verbindung ergab:

I. $0.3906 \mathrm{~g}$ Substanz gaben $0.8627 \mathrm{~g}$ Kohlensäure und $0 \cdot 1514 g$ Wasser.

II. $0.409 g$ Substanz gaben $0.9118 g$ Kohlensäure und $0 \cdot 1594 \mathrm{~g}$ Wasser.

In 100 Theilen:

$$
\begin{array}{crr}
\text { I. } & \multicolumn{1}{c}{\text { I. }} & \multicolumn{1}{c}{\mathrm{C}_{7} \mathrm{H}_{6} \mathrm{O}_{3}} \\
\mathrm{C} \ldots \ldots 60 \cdot 21 & 60 \cdot 80 & 60 \cdot 86 \\
\mathrm{H} \ldots \ldots .4 \cdot 30 & 4 \cdot 32 & 4 \cdot 34 .
\end{array}
$$

Die Substanz enthält ein Molekúl Wasser, wie nachstehende Bestimmungen ergeben:

1 Annalen der Chem. und Pharm., 134, 278. 
I. $0 \cdot 4416 \mathrm{~g}$ Substanz gaben $0 \cdot 0510 \mathrm{~g}$ Wasser. II. $0.4633 g$ Substanz gaben $0.0543 g$ Wasser.

In 100 Theilen:

$$
\begin{array}{ccc}
\text { I. } & \text { II. } & \mathrm{C}_{7} \mathrm{H}_{8} \mathrm{O}_{3}+\mathrm{H}_{2} \mathrm{O} \\
\text { Wasser } \ldots . .11 .54 & 11.72 &
\end{array}
$$

Ein Theil des Gemenges der beiden Säuren wurde in Wasser gelöst, mit Bromwasser versetzt, wodurch ein sehr reichlicher gelber Niederschlag erhalten wurde, der abgesaugt und mit verdünntem Alkohol umkrystallisirt wurde. Man erbielt so eine weisse verfilzte Masse, welche bei $91^{\circ}$ schmolz und alle Eigenschaften des Tribromphenols $\mathrm{C}_{6} \mathrm{H}_{3} \mathrm{Br}_{3} \mathrm{O}$ besass.

Die Brombestimmung der im Vacuum getrockneten Substanz ergab:

$0 \cdot 3342 g$ Substanz gaben $0.5677 g$ Bromsilber.

In 100 Theilen:

$$
\text { Br......72.29 } \frac{\mathrm{C}_{6} \mathrm{H}_{3} \mathrm{Br}_{3} \mathrm{O}}{72 \cdot 50 .}
$$

Es dürfte also wohl kaum mehr einem Zweifel unterliegen, dass eine der bei der Kalischmelze erhaltenen Säuren Paraoxybenzoësäure ist, zumal sie auch noch das von Hla siwetz und Barth ${ }^{1}$ angegebene charakteristische Bleisalz gibt.

Das Filtrat von dem flockigen Bromproduct wurde mit Natriumamalgam entbromt, mit Schwefelsäure angesänert und mit Äther ausgeschüttelt. Man erbielt auf diese Weise eine Substanz, welche die Eigenschaften der Protocatechusäure besass.

Aus $160 \mathrm{~g}$ Harz wurden etwa $10 \mathrm{~g}$ des Gemenges von Protocatechusäure and Paraoxybenzoësäure erhalten.

Zum Schlusse möge es mir gestattet sein, den Herren Professoren R. Benedikt und J. Wiesner für die freundliche Unterstützung, die sie mir bei Ausführung dieser Arbeit zu Theil werden liessen, meinen wärmsten Dank auszusprechen.

1 Annalen der Chem. und Pharm., 134, 273. 